hep-ph/0202143

DPNU-02-03

\title{
The neutrinoless double $\beta$ decay and the neutrino mass hierarchy
}

\author{
Naoyuki Haba ${ }^{1,2 *}$, and Tomoharu Suzuki ${ }^{2 \dagger}$ \\ ${ }^{1}$ Faculty of Engineering, Mie University, Tsu Mie 514-8507, Japan \\ ${ }^{2}$ Department of Physics, Nagoya University, Nagoya, 464-8602, Japan
}

\begin{abstract}
Recently the evidence of the neutrinoless double $\beta(0 \nu \beta \beta)$ decay has been announced. This means that neutrinos are Majorana particles and their mass hierarchy is forced to certain patterns in the diagonal basis of charged lepton mass matrix. We estimate the magnitude of $0 \nu \beta \beta$ decay in the classification of the neutrino mass hierarchy patterns as Type A, $m_{1,2} \ll m_{3}$, Type B, $m_{1} \sim m_{2} \gg m_{3}$, and Type C, $m_{1} \sim m_{2} \sim m_{3}$, where $m_{i}$ is the $i$-th generation neutrino absolute mass. The data of $0 \nu \beta \beta$ decay experiment suggests the neutrino mass hierarchy pattern should be Type $\mathrm{B}$ or $\mathrm{C}$. Type $\mathrm{B}$ predicts a small magnitude of $0 \nu \beta \beta$ decay which is just edge of the allowed region of experimental value in $95 \%$ c.l., where Majorana $C P$ phases should be in a certain parameter region. Type $\mathrm{C}$ can induce the suitably large amount of $0 \nu \beta \beta$ decay which is consistent with the experimental data, where overall scale of degenerate neutrino mass plays a crucial role, and its large value can induce the large $0 \nu \beta \beta$ decay in any parameter regions of Majorana $C P$ phases.
\end{abstract}

*haba@eken.phys.nagoya-u.ac.jp

${ }^{\dagger}$ tomoharu@eken.phys.nagoya-u.ac.jp 


\section{Introduction}

Recently the evidences of neutrino oscillations are strongly supported by both of the atmospheric [1, 2] and the solar neutrino experiments [3, 4, 5, 6]. The former suggests an almost maximal lepton flavor mixing between the 2 nd and the $3 \mathrm{rd}$ generations, while the favorable solution to the solar neutrino deficits is given by large mixing angle solution between the 1st and the 2nd generations ( LMA, LOW or VO ) [7]. Neutrino oscillation experiments indicate that the neutrinos have tiny but finite masses, with two mass squared differences $\Delta m_{\odot}^{2}<\Delta m_{\mathrm{atm}}^{2}$. However, we cannot know the absolute values of the neutrinos masses from the oscillation experiments.

Recently, a paper [8] announces the evidence of the neutrinoless double $\beta(0 \nu \beta \beta)$ decay. This paper suggests

$$
\langle m\rangle=(0.05-0.86) \mathrm{eV} \quad \text { at } 95 \% \text { c.l. } \quad \text { (best value } 0.4 \mathrm{eV}) \text {. }
$$

This results is very exciting. It is because $0 \nu \beta \beta$ decay experiments could tell us about the absolute value of the neutrino masses, while neutrino oscillation experiments show only mass squared differences of neutrinos. The evidence for $0 \nu \beta \beta$ decay also means neutrinos are the Majorana particles and the lepton number is violated, since $0 \nu \beta \beta$ decay cannot occur in the case of Dirac neutrinos. This evidence is also closely related to the recent topics of the cosmology such as the dark matter candidate of universe [9].

The tiny neutrino masses and the lepton flavor mixings have been discussed in a lot of models beyond the Standard Model (SM). One of the most promising ideas is that light neutrinos are constructed as Majorana particles in the low energy, such as the see-saw mechanism [10]. Here we are concentrating on the light Majorana neutrinos which masses are induced by the dimension five operators in the low energy effective Yukawa interactions.

In this paper, we will estimate the magnitude of $0 \nu \beta \beta$ decay in the classification of the neutrino mass hierarchy patterns as Type A, $m_{1,2} \ll m_{3}$, Type B, $m_{1} \sim m_{2} \gg m_{3}$, and Type C, $m_{1} \sim m_{2} \sim m_{3}$, where $m_{i}$ is the $i$-th generation neutrino absolute mass [11]. The magnitude of $0 \nu \beta \beta$ decay strongly depends on the neutrino mass hierarchy. We will analyze the value of $\langle m\rangle$ in the parameter space of the Majorana $C P$ phases, and search the region of being consistent with the data of $0 \nu \beta \beta$ decay experiment.

\section{Neutrino masses and flavor mixings}

Before starting our discussions, let us show the notations of neutrino masses and their mixing angles. Since we would like to be concentrating on the low energy mass matrix of Majorana neutrinos, we add the dimension five operator to the standard model in the 
lepton sector as,

$$
\mathcal{L}=\left(Y_{e}\right)_{i j} L_{i} E_{j} H_{1}+\kappa_{i j}\left(L_{i} H_{2}\right)\left(L_{j} H_{2}\right)
$$

where $\kappa_{i j}$ is the coefficient of the dimension five operator, and $L_{i}$ and $E_{i}$ are $i$-th generation lepton doublet and charged lepton singlet with $(i=1 \sim 3)$, respectively, $H_{1}$ and $H_{2}$ are Higgs doublets $\$$. The light neutrinos obtain masses from the vacuum expectation value $(\mathrm{VEV})$ of $v_{2}=\left\langle H_{2}\right\rangle$ as

$$
\left(M_{\nu}\right)_{i j}=\kappa_{i j} v_{2}^{2}
$$

The energy scale of $\kappa_{i j}^{-1}$ suggests the scale of the new physics and the lepton number violation. In the diagonal base of the charged lepton sector, the light neutrino mass matrix, $\left(M_{\nu}\right)_{i j}$, is diagonalized by $U_{i j}$ as

$$
U^{\mathrm{T}} M_{\nu} U \equiv M_{\nu}^{\text {diag }}=\operatorname{diag}\left(m_{1}, m_{2}, m_{3}\right) .
$$

Here the matrix $U$ is so-called MNS matrix 12 denoted by

$$
U=V \cdot P
$$

where $V$ is the CKM-like matrix, which contains one $C P$-phase $(\delta)$,

$$
V=\left(\begin{array}{ccc}
c_{13} c_{12} & c_{13} s_{12} & s_{13} e^{-i \delta} \\
-c_{23} s_{12}-s_{23} s_{13} c_{12} e^{i \delta} & c_{23} c_{12}-s_{23} s_{13} s_{12} e^{i \delta} & s_{23} c_{13} \\
s_{23} s_{12}-c_{23} s_{13} c_{12} e^{i \delta} & -s_{23} c_{12}-c_{23} s_{13} s_{12} e^{i \delta} & c_{23} c_{13}
\end{array}\right)
$$

and $P$ contains two extra Majorana phases $\left(\phi_{1,2}\right)$,

$$
P=\operatorname{diag} \cdot\left(e^{-i \phi_{1} / 2}, e^{-i \phi_{2} / 2}, 1\right)
$$

For the latter convenience, we introduce the matrix

$$
\widetilde{M}_{\nu} \equiv P^{*} M_{\nu}^{\text {diag }} P^{*}=\operatorname{diag} \cdot\left(m_{1} e^{i \phi_{1}}, m_{2} e^{i \phi_{2}}, m_{3}\right)=\operatorname{diag} \cdot\left(\tilde{m}_{1}, \tilde{m}_{2}, \tilde{m}_{3}\right)
$$

Table 1 shows the results from the recent neutrino oscillation experiments [1, 2, 3, 4, 5, 6, 7]. These results indicate that the neutrinos have tiny but finite masses, with two mass squared differences $\Delta m_{\odot}^{2}<\Delta m_{\text {atm }}^{2}$. The naive explanation of the present neutrino oscillation experiments is that the solar neutrino anomaly is caused by the mixings of the 1st and the 2nd generations $\left(\theta_{\odot} \simeq \theta_{12}, \Delta m_{\odot}^{2} \simeq m_{2}^{2}-m_{1}^{2}\right)$, and atmospheric neutrino deficit is caused by the mixings of the 2 nd and the 3 rd generations $\left(\theta_{\text {atm }} \simeq \theta_{23}, \Delta m_{\text {atm }}^{2} \simeq m_{3}^{2}-m_{2}^{2}\right)$. Considering the results of the oscillation experiments, the hierarchical patterns of neutrino masses are classified by the following three types:

* Although we write Yukawa interactions in Eq. 26 by the supersymmetric forms, the results in this paper do not depend on whether the model is supersymmetric or not. 


\begin{tabular}{||c||cc||}
\hline \hline Solar & $\Delta m_{\odot}^{2}\left(\mathrm{eV}^{2}\right)$ & $\sin ^{2} 2 \theta_{\odot}$ \\
\hline SMA & $(4-9) \times 10^{-6}$ & $(0.0008-0.008)$ \\
LMA & $(2-20) \times 10^{-5}$ & $(0.3-0.93)$ \\
LOW & $(6-20) \times 10^{-8}$ & $(0.89-1)$ \\
VO & $10^{-10}$ & $(0.7-0.95)$ \\
Just So & $(5-8) \times 10^{-12}$ & $(0.89-1)$ \\
\hline \hline Atmospheric & $\Delta m_{\text {atm }}^{2}\left(\mathrm{eV}^{2}\right)$ & $\sin ^{2} 2 \theta_{\text {atm }}$ \\
\hline & $(1.8-4.0) \times 10^{-3}$ & $(0.87-1)$ \\
\hline \hline
\end{tabular}

Table 1: The allowed values of $\Delta m_{\odot}^{2}, \Delta m_{a t m}^{2}, \sin ^{2} 2 \theta_{\odot}$, and $\sin ^{2} 2 \theta_{a t m}$ from the neutrino oscillation experiments [1, 2, 63, 因, 5, 6, 王.

$$
\begin{array}{ll}
\mathrm{A} & : \quad m_{3} \gg m_{1,2} \\
\mathrm{~B} & : \quad m_{1} \sim m_{2} \gg m_{3} \\
\mathrm{C} \quad: \quad m_{1} \sim m_{2} \sim m_{3} .
\end{array}
$$

Taking into account of the mass squared differences, $\Delta m_{\odot}^{2}$ and $\Delta m_{\text {atm }}^{2}$, the absolute masses of the neutrino in the leading are written by

$\underline{\text { Type A }}$

$$
\begin{array}{lll}
m_{1} & : & 0 \\
m_{2} & : & \sqrt{\Delta m_{\odot}^{2}} \\
m_{3} & : & \sqrt{\Delta m_{a t m}^{2}}
\end{array}
$$

Type C

$$
\begin{array}{ll}
m_{1}: & m_{\nu} \\
m_{2}: & m_{\nu}+\frac{1}{2} \frac{\Delta m_{\odot}^{2}}{m_{\nu_{2}}} \\
m_{3}: & m_{\nu}+\frac{1}{2} \frac{\Delta m_{a t m}^{2}}{m_{\nu}},
\end{array}
$$

Type B

$$
\begin{array}{lll}
m_{1}: & \sqrt{\Delta m_{a t m}^{2}} \\
m_{2}: & \sqrt{\Delta m_{a t m}^{2}}+\frac{1}{2} \frac{\Delta m_{\odot}^{2}}{\sqrt{\Delta m_{a t m}^{2}}} \\
m_{3}: & 0
\end{array}
$$

in each type, respectively. Where $m_{\nu}$ in Type $\mathrm{C}$ is the scale of the degenerated neutrino masses.

Table 1 shows the flavor mixing angles in the lepton sector. The mixing angle between the 2 nd and the 3rd generations is almost maximal mixing from the atmospheric neutrino experiments. There are three candidates [ of solutions for solar neutrino problems. Recent super-kamiokande data of day/night effects implies the LMA solution are most favorable solution [3]. We use the center values of the mixing angles $\theta_{12}$ and $\theta_{23}$,

\footnotetext{
${ }^{\dagger}$ Since there are no defferences between the three solutions LOW, VO and Just So, we consider them together VAC solution.
} 


$$
\begin{array}{ll}
\Delta m_{a t m}^{2}=3.2 \times 10^{-3} \mathrm{eV}^{2}, & \Delta m_{\odot}^{2}=4.5 \times 10^{-5} \mathrm{eV}^{2}, \\
\sin ^{2} 2 \theta_{a t m}=1.0, & \tan ^{2} \theta_{\odot}=4.1 \times 10^{-1},
\end{array}
$$

in the following numerical analyses. The rest of the mixing angle, $\theta_{13}$, is not measured as the deficit value, the upper bound is given as,

$$
\sin ^{2} 2 \theta_{13}<0.1
$$

from the CHOOZ experiments[13].

In the zeroth order approximations, $\cos \theta_{12}=\cos \theta_{23}=1 / \sqrt{2}$ and $\sin \theta_{13}=0$, we can obtain the zeroth order form of the MNS matrixf as,

$$
V^{(0)}=\left(\begin{array}{ccc}
\frac{1}{\sqrt{2}} & \frac{1}{\sqrt{2}} & 0 \\
-\frac{1}{2} & \frac{1}{2} & \frac{1}{\sqrt{2}} \\
\frac{1}{2} & -\frac{1}{2} & \frac{1}{\sqrt{2}}
\end{array}\right)
$$

The neutrino mass matrix $M_{\nu}$ is determined by $U$ and $M_{\nu}^{\text {diag }}$ from Eqs.(雨) (6). The zeroth order form of the neutrino mass matrix is determined by the approximated MNS matrix, $V$, according to the patterns of neutrino mass hierarchy, Types $A \sim C$. In Ref.[11], the zeroth order forms of neutrino mass matrices are shown when Majorana $C P$ phases are 0 or $\pi$, which are shown in Table 2. These mass matrices are useful for the first approximations of estimating the probability of $0 \nu \beta \beta$. We use continuous values of Majorana $C P$ phases in the following analyses.

\section{$3 \quad$ Neutrino mass hierarchies and $0 \nu \beta \beta$ decay}

Now we are in a position of discussing the relations between the neutrino mass hierarchy patterns and the magnitude of $0 \nu \beta \beta$ decay. The effective neutrino mass $\langle m\rangle$, which shows the magnitude of $0 \nu \beta \beta$ decay in Eq.(1) is defined by

$$
\langle m\rangle=\left|\sum_{i=1}^{3} U_{e i}^{2} m_{i}\right|=\left|\sum_{i=1}^{3} U_{e i} m_{i} U_{i e}^{\mathrm{T}}\right|=\left|V_{e 1}^{2} m_{1} e^{i \phi_{1}}+V_{e 2}^{2} m_{2} e^{i \phi_{2}}+V_{e 3}^{2} m_{3}\right|,
$$

where $i$ denotes the label of the mass eigenstate $(i=1,2,3)$. What pattern of neutrino mass hierarchy can induce the sizeable amount of $0 \nu \beta \beta$ decay amplitude in Eq.(目)? In the

$\ddagger$ The deviation from this zeroth order approximation is important. As will be shown in the next section, the tiny difference between the maximal and the LMA angle causes the sensitive effects to the mass neutrino matrix. 
approximation in the previous section, the value of $\langle m\rangle$ is equal to that of $(1,1)$ component of $\widetilde{M}_{\nu}$. Thus, Table 2 suggests that the forms of the neutrino mass matrix should be B2 or $\mathrm{C} 0$ or $\mathrm{C} 3$, in order to obtain the suitable large magnitude of $(1,1)$ component. However, it is too naive estimation. We will estimate the magnitude of $\langle m\rangle$ more accurately, which patterns of neutrino mass hierarchies are really consistent with $0 \nu \beta \beta$ decay data in Eq.(11) within the fluctuations of physical parameters of a tiny value of $\left|U_{e 3}\right|\left(=\sin \theta_{13}\right)$, CKM-like phase $\delta$ and Majorana phases $\phi_{1,2}$. We will show the analyses according to the neutrino mass hierarchy patterns, Type $\mathrm{A} \sim \mathrm{C}$ in the base of Majorana $C P$ phases.

\section{$3.1 \quad$ Type A}

Equation (9) suggests the largest value of $\langle m\rangle$ is given by ,

$$
\langle m\rangle_{\mathrm{MAX}}=\left|V_{e 1}\right|^{2} m_{1}+\left|V_{e 2}\right|^{2} m_{2}+\left|V_{e 3}\right|^{2} m_{3}
$$

In the Type A mass hierarchy, the main contribution to $\langle m\rangle$ is 2nd term or 3rd term of Eq.(10) depending on the magnitude of $V_{e 3}$. However, both terms induce $\mathcal{O}\left(10^{-3}\right) \mathrm{eV}$ contribution to the value of $\langle m\rangle$ within the fluctuations of physical parameters, $V_{e 3}$. Even

if $m_{1}$ in Eq.(10) is lifted as $m_{1} \sim \sqrt{\Delta m_{\odot}^{2}} \leq m_{2}$, the magnitude of $\langle m\rangle$ is still less than of $\mathcal{O}\left(10^{-2}\right) \mathrm{eV}$. These magnitudes are too small to explain the recent $0 \nu \beta \beta$ decay experiments in Eq.(11). Thus we can conclude that the Type A neutrino mass hierarchy pattern can not explain the $0 \nu \beta \beta$ results in Eq.(1).

\section{$3.2 \quad$ Type B}

Next, let us see the case of Type B. When Majorana $C P$ phases take the specific values of 0 or $\pi$, Type B is devided into two cases B1 and B2, which are shown in Table 2.

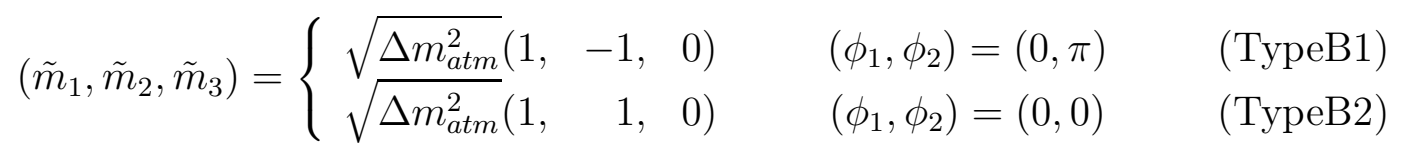

Where mass eigenvalues show the zeroth order values. The sign of $m_{1}$ is the opposite (same) as that of $m_{2}$ in Type B1 (B2). Table 2 shows that B2 induces $\langle m\rangle=\mathcal{O}\left(\sqrt{\Delta m_{\text {atm }}^{2}}\right)$, while that of $\mathrm{B} 1$ does not in the zeroth order. Thus we can expect the sizeable amplitude of $0 \nu \beta \beta$ decay in B2, not B1. Since the Majorana phases, $\phi_{1,2}$, connect B1 and B2 continuously, we estimate the value of $\langle m\rangle$ with nontrivial Majorana phases, and see also the effects of other small quantities such as $U_{e 3}$.

At first, we estimate the effect of the tiny difference of LMA mixing angle $\theta_{\odot}$ from the maximal mixing between the 1 st and the 2 nd generations. The recent oscillation 


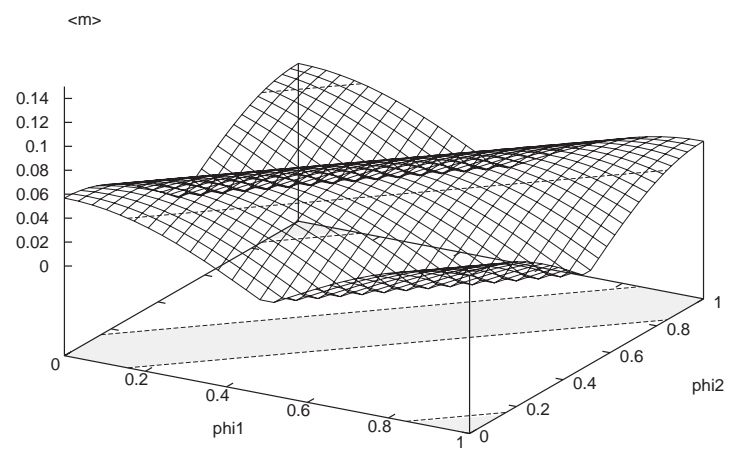

(a)

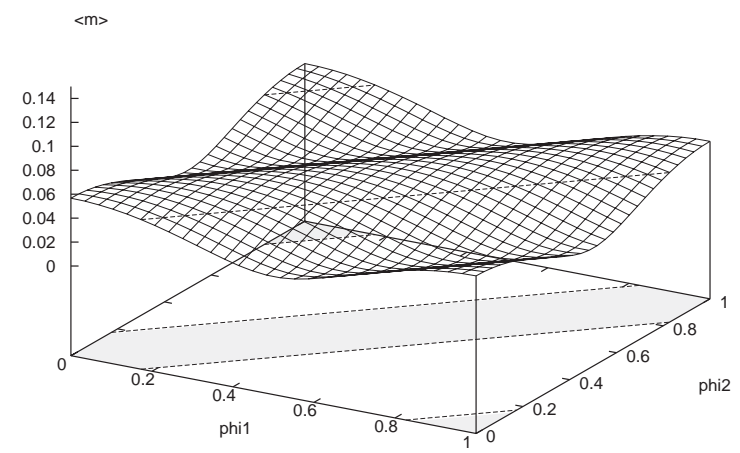

(b)

Figure 1: The values of $\langle m\rangle$ with the 1-2 maximal mixing, $\tan \theta_{\odot}=0$, in (a), and the LMA center value, $\tan \theta_{\odot}=2.6 \times 10^{-1}$, in (b). Other values are taken as the zeroth order values as $U_{e 3}=0$ and $\theta_{a t m}=\pi / 4$. Majorana phases $\phi_{1,2}$ are normalized by $2 \pi$, and the lines $\phi_{1}-\phi_{2}=n+1 / 2\left(\phi_{1}-\phi_{2}=n\right)$ correspond to Type B1 (B2) where $n=0, \pm 1, \pm 2 \cdots$. The region of the large value of $\langle m\rangle$ satisfying Eq.(四) is shown as the grayed drown region.

experiments show the LMA solution disfavor the complete maximal mixing. Figure [1 shows the values of $\langle m\rangle$ with the 1-2 maximal mixing, $\tan \theta_{\odot}=0$ in (a), and the LMA center value, $\tan \theta_{\odot}=2.6 \times 10^{-1}$, in (b). Other values are taken as $U_{e 3}=0$ and $\theta_{\text {atm }}=\pi / 4$. The lines where $\phi_{1}-\phi_{2}=(2 n+1) \pi\left(\phi_{1}-\phi_{2}=2 n \pi\right)$ correspond to Type B1 (B2) where $n=0, \pm 1, \pm 2 \cdots$. Only one degree of freedom, $\phi_{1}-\phi_{2}$, is physical since $m_{3}=0$ in Type B (see Eq.(6)). The region of the large value of $\langle m\rangle$ satisfying Eq.(11) is shown as the grayed drown region. Apparently only the region around B2 can satisfy Eq.(1). The wide region include B1 does not satisfy Eq.(1). Figure 11 shows that Majorana phases connect B1 and B2 continuously. Comparing to (b) with (a) in Fig.1, we find that the small change from the maximal mixing, makes an allowed region, where the value $\langle m\rangle$ is larger than $0.05 \mathrm{eV}$, be spreaded. Especially for the values of $\langle m\rangle$, although they are almost zero in the region around Type B2 in (a), they become significantly enhanced in the same region in (b). This means that the tiny deviation of $\theta_{\odot}$ from the zeroth order classification of neutrino mass matrices, in Table 2, is important. It is because the accidental cancellation between $U_{e 1}$ and $U_{e 2}$ induced from two maximal mixings, $\theta_{\odot}=\theta_{a t m}=\pi / 4$, in (a) is forbidden in (b). In the following analyses we use the $\theta_{\odot}=2.6 \times 10^{-1}$ as LMA angle, not maximal mixing.

Next, we see the effect of $U_{e 3}$ in Type B. In general the change of $U_{e 3}$ induces the changes of $U_{e 1}$ and $U_{e 2}$, which causes the deviation of $\langle m\rangle$ in Eq.(9). Since we only know the upper bound of $\left|U_{e 3}\right|$ as, $\left|U_{e 3}\right|<0.16$, we compare the cases of $\left|U_{e 3}\right|=0$ in Fig.2 (a), and $\left|U_{e 3}\right|=0.1$ in Fig.2 (b). The value of $\langle m\rangle$ becomes small as the magnitude of $U_{e 3}$ 


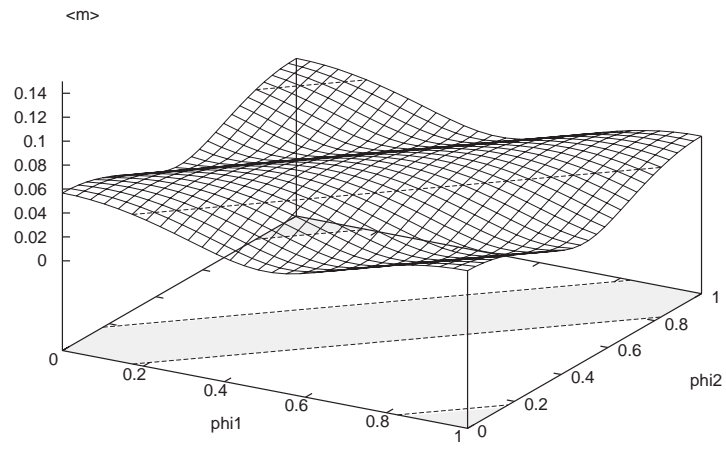

(a)

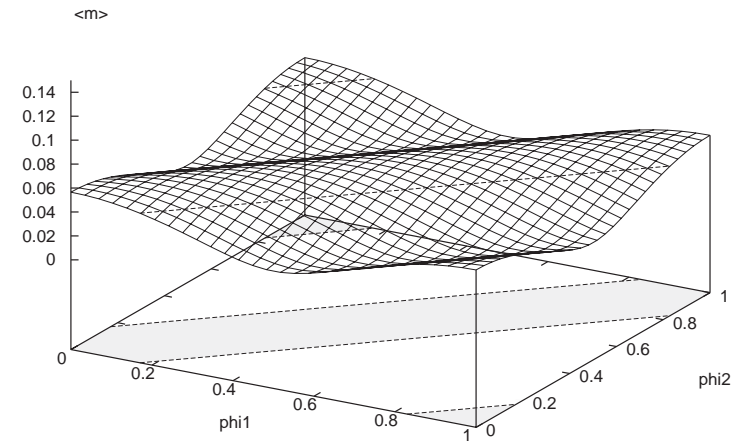

(b)

Figure 2: The values of $\langle m\rangle$ with $\left|U_{e 3}\right|=0$ (a) and $\left|U_{e 3}\right|=0.1$ (b). Other parameters are set as $\tan \theta_{\odot}=2.6 \times 10^{-1}$ and $\theta_{\text {atm }}=\pi / 4$. Majorana phases $\phi_{1}$ and $\phi_{2}$ are normalized by $2 \pi$.

becomes large. However, this effect is negligibly small shown in Fig.2 (a) and (b). So the effect of the magnitude of $U_{e 3}$ to $\langle m\rangle$ is negligible. The crucial point of this result comes from $m_{3} \simeq 0$ in Eq.(9). In the case of non-zero value of $U_{e 3}, C P$ phase $\delta$ is physical, but this effect is also negligible.

We also estimete the effect of the deviation from $m_{3}=0$. Since $m_{3}$ should be much smaller than the value of $\left|m_{1}\right| \simeq 5.6 \times 10^{-2} \mathrm{eV}$ in Type $\mathrm{B}$, we take $m_{3}=0.1 \times m_{1}$. We find that this effect is also negligible.

As shown above, the values of $\langle m\rangle$ cannot be larger than $0.06 \mathrm{eV}$ in any parameter sets in Type B. The region where $\langle m\rangle>0.05 \mathrm{eV}$ only exists just around B2. This magnitude of $\langle m\rangle$ is just edge of the allowed region of experimental value of $95 \%$ c.l. in Eq.(1), and far from the best fit value, $0.4 \mathrm{eV}$. The improvement of experiments might dispose the possibility of type B in the near future.

\subsection{Type C}

The neutrino masses are degenerate in Type $\mathrm{C}$, and we set the value of the degenerate mass as $m_{\nu}$. In Ref. 11], Type $\mathrm{C}$ mass hierarchy is classified to four subgroups, C0, C1, C2 and $\mathrm{C} 3$, by relative signs $m_{1}, m_{2}$ and $m_{3}$.

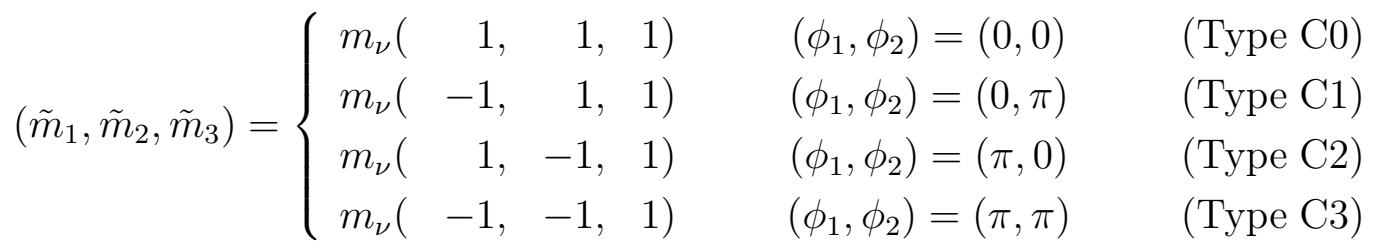




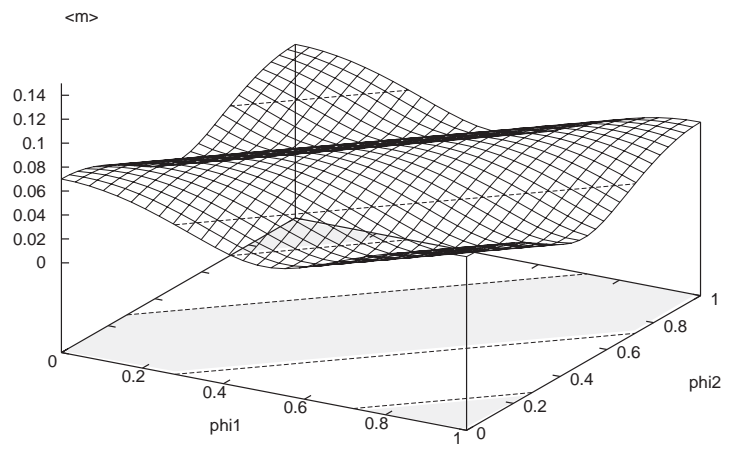

(a)

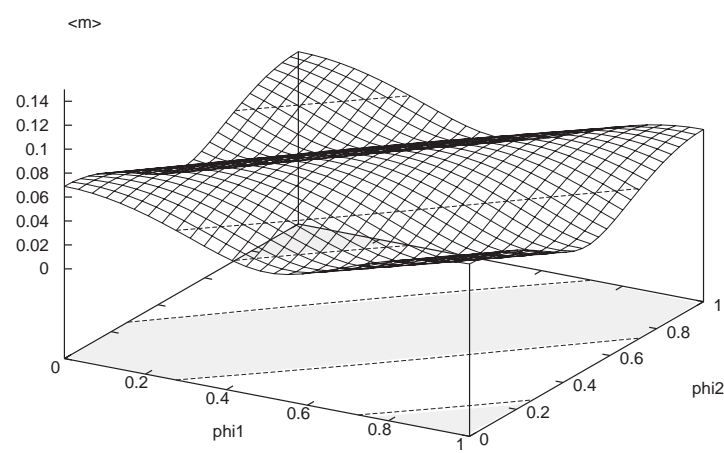

(c)

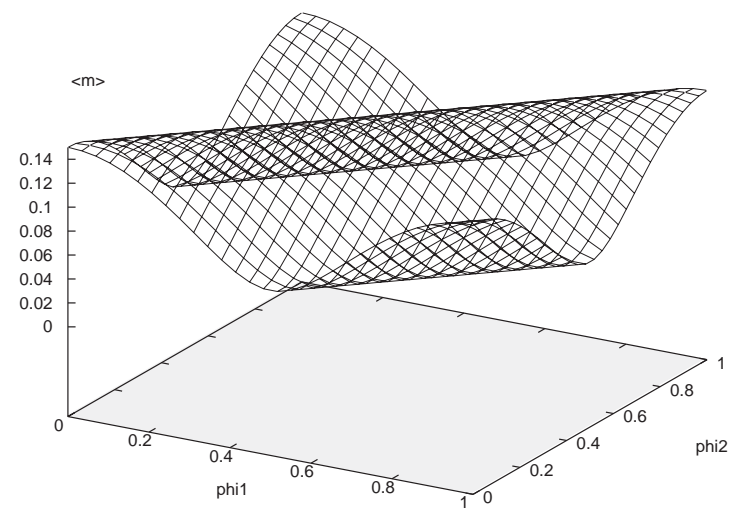

(b)

Figure 3: The values of $\langle m\rangle$ in Type C. Each graph shows (a): $m_{\nu}=0.07 \mathrm{eV}, U_{e 3}=0$, (b): $m_{\nu}=0.15 \mathrm{eV}, U_{e 3}=0$ and (c): $m_{\nu}=0.07 \mathrm{eV},\left|U_{e 3}\right|=0.1, \delta=\pi / 2$. Other parameters are set as $\tan \theta_{\odot}=2.6 \times 10^{-1}$ and $\theta_{\text {atm }}=\pi / 4$. Majorana phases $\phi_{1,2}$ are normalized by $2 \pi$.

Seeing the zeroth order neutrino mass matrices in Table ₹, we suppose naively only Type $\mathrm{C} 0$ and $\mathrm{C} 3$ might explain $0 \nu \beta \beta$ decay experiments because the $(1,1)$ element of mass matrix is of $\mathcal{O}\left(m_{\nu}\right)$. Can Type $\mathrm{C} 1$ and $\mathrm{C} 2$ not really explain Eq.(四)?

In Fig. 3 (a) and Fig. (b) (b), the values of $\langle m\rangle$ are shown in the case of $m_{\nu}=0.07 \mathrm{eV}$ and $m_{\nu}=0.15 \mathrm{eV}$, respectively. Where we take $U_{e 3}=0$. It is worth noting that the values of $\langle m\rangle$ strongly depend on the value of $m_{\nu}$. In the case of $m_{\nu}=0.15 \mathrm{eV}$ in Fig. 3 (b), any values of $\phi_{1,2}$ can explain the $0 \nu \beta \beta$ decay in Eq.(1]). The suitably large value of $\langle m\rangle$ satisfying Eq.(1) can be obtained in Type $\mathrm{C} 1$ and $\mathrm{C} 2$, although zeroth order forms of neutrino mass matrices in Table 2 show tiny $(1,1)$ elements in Type $\mathrm{C} 1$ and $\mathrm{C} 2$. This is simply because that $\theta_{\odot}$ deviates from the maximal mixing tinily and that all elements, of course including the $(1,1)$ component, become large as $m_{\nu}$ becomes large. Thus the suitable large $\langle m\rangle$ can be obtained even if the $(1,1)$ component is of order zero in the 
zeroth order approximation in Table 2.

As for the effects of $\left|U_{e 3}\right|$ and $C P$ phase $\delta$, they should induce the sensitive effects to the magnitude of $\langle m\rangle$ in Type $\mathrm{C}$, because the value of $m_{3}$ is large comparing to Type $\mathrm{B}$. We show the case of $C P$ phase $\delta=\pi / 2$ (Fig. 3 (c)), where we take $\left|U_{e 3}\right|=0.10$ and $m_{\nu}=0.07$ eV. From Figs. 3 (a) and (c), we find that the region around $\left(\phi_{1}, \phi_{2}\right)=(\pi, \pi)$ receives the sensitive effects from $\left|U_{e 3}\right|$ and $\delta$, but they are not so large. We also estimate the effects in the case of $\delta=0$, but we find that this effect is negligible.

As shown above, the main contribution which lifts the value of $\langle m\rangle$ exists in $m_{\nu}$ in Type C. Thus we can conclude that the suitably large $m_{\nu}$ makes the value of $\langle m\rangle$ be large enough to satisfy Eq.(1) in any values of Majorana phases $\phi_{1}$ and $\phi_{2}$ in Type C.

\section{Conclusion}

A recent paper [8] announces the evidence of $0 \nu \beta \beta$ decay, and the value of $\langle m\rangle$ is large as Eq.(1). In this paper, we have estimated the value of $\langle m\rangle$ according to the neutrino mass hierarchy patterns, Type A, B and C. We have searched which mass hierarchy is consistent with the recent $0 \nu \beta \beta$ decay experiments. We have also analyzed the deviation from the zeroth order forms of neutrino mass matrices with the fluctuations of small physical parameters in the base of the Majorana $C P$ phases.

The results are the followings. Type A cannot explain the $0 \nu \beta \beta$ results in Eq.(1). In Type B, the region where $\langle m\rangle>0.05 \mathrm{eV}$ exists around $\mathrm{B} 2$ in the parameter space of Majorana $C P$ phases. In Type $\mathrm{C}$, the suitably large $m_{\nu}$ makes the value of $\langle m\rangle$ be large enough to satisfy Eq.(1) in any values of Majorana phases $\phi_{1}$ and $\phi_{2}$, contrary to the zeroth

order estimations. The effects of $\left|U_{e 3}\right|$ and $\delta$ are not be significant in all types of neutrino mass hierarchies.

\section{Acknowledgment}

We would like to thank M. Tanimoto for letting authors know the papaer of neutrinoless double $\beta$. This work is supported in part by the Grant-in-Aid for Science Research, Ministry of Education, Science and Culture, Japan (No. 12740146).

\section{References}

[1] Y. Fukuda et al. [Kamiokande Collaboration], Phys. Rev. Lett. 77 (1996) 1683.

[2] Y. Fukuda et al. [Kamiokande Collaboration], Phys. Lett. B 335 (1994) 237;

Y. Fukuda et al. [Super-Kamiokande Collaboration], Phys. Rev. Lett. 81 (1998) 1562; 
T. Kajita [Super-Kamiokande Collaboration], in Neutrino Physics and Astrophysics, Proceedings of the XVIIIth International Conference on Neutrino Physics and Astrophysics (Neutrino '98), June 4-9, 1998, Takayama, Japan, edited by Y. Suzuki and Y. Totsuka, (Elsevier Science B.V., Amsterdam, 1999) page 123; Nucl. Phys. Proc. Suppl. 77, 123 (1999).

[3] Y. Fukuda et al. [Super-Kamiokande Collaboration],

Phys. Rev. Lett. 81 (1998) 1158; Erratum-ibid. 81 (1998) 4279;

Phys. Rev. Lett. 82 (1999) 2430;

Phys. Rev. Lett. 82 (1999) 1810.

[4] Q. R. Ahmad et al. [SNO Collaboration], Phys. Rev. Lett. 87, 071301 (2001).

[5] K. Lande et al., Astrophys. J. 496 (1998) 505.

[6] V. N. Gavrin [SAGE Collaboration], Nucl. Phys. Proc. Suppl. 91 (2001) 36;

E. Bellotti, Nucl. Phys. Proc. Suppl. 91 (2001) 44.

[7] V. D. Barger, D. Marfatia and K. Whisnant, Phys. Rev. Lett. 88, 011302 (2002); G. L. Fogli, E. Lisi, D. Montanino and A. Palazzo, Phys. Rev. D 64, 093007 (2001); J. N. Bahcall, M. C. Gonzalez-Garcia and C. Pena-Garay, JHEP 0108, 014 (2001); A. Bandyopadhyay, S. Choubey, S. Goswami and K. Kar, Phys. Lett. B 519, 83 (2001).

[8] H. V. Klapdor-Kleingrothaus, A. Dietz, H. L. Harney and I. V. Krivosheina, Mod. Phys. Lett. A 16, 2409 (2002)

[9] V. Barger, S. L. Glashow, D. Marfatia and K. Whisnant, arXiv:hep-ph/0201262.

[10] T. Yanagida, "Horizontal Symmetry And Masses Of Neutrinos", Prog. Theor. Phys. 64 (1980) 1103, and in Proceedings of the "Workshop on the Unified Theory and the Baryon Number in the Universe", Tsukuba, Japan, Feb 13-14, 1979, Eds. O. Sawada and A. Sugamoto, KEK report KEK-79-18, p. 95;

M. Gell-Mann, P. Ramond and R. Slansky, in "Supergravity" (North-Holland, Amsterdam, 1979) eds. D.Z. Freedman and P. van Nieuwenhuizen, Print-80-0576 (CERN). Phys. Lett. B 161 (1985) 141.

[11] G. Altarelli and F. Feruglio, Phys. Rept. 320, 295 (1999).

[12] Z. Maki, M. Nakagawa, and S. Sakata, Prog. Theor. Phys. 28 (1962) 870.

[13] M. Apollonio et al. [CHOOZ Collaboration], Phys. Lett. B 466, 415 (1999) 


\begin{tabular}{|c|c|c|}
\hline & $\widetilde{M}_{\nu}$ & $\begin{array}{c}\text { Neutrino } \\
\text { mass matrix }\end{array}$ \\
\hline A & $\operatorname{diag} .(0,0,1)$ & {$\left[\begin{array}{ccc}0 & 0 & 0 \\
0 & 1 / 2 & 1 / 2 \\
0 & 1 / 2 & 1 / 2\end{array}\right]$} \\
\hline B1 & $\operatorname{diag} .(1,-1,0)$ & $\begin{array}{ccc}0 & -1 / \sqrt{2} & 1 / \sqrt{2} \\
-1 / \sqrt{2} & 0 & 0 \\
1 / \sqrt{2} & 0 & 0\end{array}$ \\
\hline B2 & diag. $(1,1,0)$ & $\left.\begin{array}{ccc}1 & 0 & 0 \\
0 & 1 / 2 & -1 / 2 \\
0 & -1 / 2 & 1 / 2\end{array}\right]$ \\
\hline
\end{tabular}

\begin{tabular}{|l|c|cc|}
\hline & & {$\left[\begin{array}{lll}1 & 0 & 0 \\
0 & 1 & 0 \\
0 & 0 & 1\end{array}\right]$} \\
\hline C1 & diag. $(1,1,1)$ & diag. $(-1,1,1)$ & {$\left[\begin{array}{ccc}0 & 1 / \sqrt{2} & -1 / \sqrt{2} \\
1 / \sqrt{2} & 1 / 2 & 1 / 2 \\
-1 / \sqrt{2} & 1 / 2 & -1 / 2\end{array}\right]$} \\
\hline C2 & diag. $(1,-1,1)$ & {$\left[\begin{array}{ccc}0 & -1 / \sqrt{2} & 1 / \sqrt{2} \\
-1 / \sqrt{2} & 1 / 2 & 1 / 2 \\
1 / \sqrt{2} & 1 / 2 & 1 / 2\end{array}\right]$} \\
\hline C3 & diag.(-1,-1,1) & {$\left[\begin{array}{ccc}-1 & 0 & 0 \\
0 & 0 & 1 \\
0 & 1 & 0\end{array}\right]$} \\
\hline & & {$\left[\begin{array}{ccc} \\
\end{array}\right.$} & \\
\hline
\end{tabular}

Table 2: The zeroth order neutrino mass matrices. In Type $\mathrm{A}$ and $\mathrm{B}$, the eigenvalues of $\widetilde{M}_{\nu}$ and the neutrino mass matricies are normalized by $\sqrt{\Delta m_{a t m}^{2}}$. In Type C, they are normalized by $m_{\nu}$. 